\title{
The structure of zonal jets in geostrophic turbulence
}

\author{
Richard K. Scott $†$ and David G. Dritschel \\ School of Mathematics and Statistics, University of St Andrews, St Andrews KY16 9SS, UK \\ (Received 26 October 2011; revised 17 July 2012; accepted 9 August 2012; \\ first published online 20 September 2012)
}

The structure of zonal jets arising in forced-dissipative, two-dimensional turbulent flow on the $\beta$-plane is investigated using high-resolution, long-time numerical integrations, with particular emphasis on the late-time distribution of potential vorticity. The structure of the jets is found to depend in a simple way on a single nondimensional parameter, which may be conveniently expressed as the ratio $L_{R h} / L_{\varepsilon}$, where $L_{R h}=\sqrt{U / \beta}$ and $L_{\varepsilon}=\left(\varepsilon / \beta^{3}\right)^{1 / 5}$ are two natural length scales arising in the problem; here $U$ may be taken as the r.m.s. velocity, $\beta$ is the background gradient of potential vorticity in the north-south direction, and $\varepsilon$ is the rate of energy input by the forcing. It is shown that jet strength increases with $L_{R h} / L_{\varepsilon}$, with the limiting case of the potential vorticity staircase, comprising a monotonic, piecewise-constant profile in the north-south direction, being approached for $L_{R h} / L_{\varepsilon} \sim O(10)$. At lower values, eddies created by the forcing become sufficiently intense to continually disrupt the steepening of potential vorticity gradients in the jet cores, preventing strong jets from developing. Although detailed features such as the regularity of jet spacing and intensity are found to depend on the spectral distribution of the forcing, the approach of the staircase limit with increasing $L_{R h} / L_{\varepsilon}$ is robust across a variety of different forcing types considered.

Key words: geostrophic turbulence, quasi-geostrophic flows, turbulent mixing

\section{Introduction}

Large-scale zonal (longitudinally aligned) jets are observed in a wide range of geophysical flows including those of the terrestrial atmosphere and oceans and the atmospheres of the gas giant planets. They coexist with a background of inhomogeneous turbulent motions and the relative intensity of the jets to the turbulent background has a strong influence on the meridional transport of important quantities such as heat, momentum, and constituent tracers, the latter including chemically and thermodynamically important quantities such as ozone and water vapour. The interplay between the jets and the turbulent background is highly complex, turbulent motions being organized into latitudinal bands by the jets, the jets themselves being maintained against dissipation via eddy momentum fluxes (see e.g. the recent review by McIntyre 2008, and references therein).

The fact that jets are observed in such a wide range of dynamical regimes, and in the presence of very different forcing mechanisms, suggests that robust dynamical 
processes are involved in their generation. It has been argued, for example, that the strong jets observed in the Jovian atmosphere are dynamically similar to the much weaker jets observed in the oceans (Galperin et al. 2004). However, beyond the conclusion that the jets are driven by eddy momentum fluxes in each case, the similarities and differences are not well-understood. In particular, it is unclear what controls fundamental properties such as the intensity, spacing, and steadiness of the jets in these cases.

Regarding the spacing of the jets, it has long been understood that it is closely linked to a fundamental length scale of the flow, the Rhines scale, given by $L_{R h}=\sqrt{U / \beta}$ (Rhines 1975; Williams 1978), where $U$ is a typical velocity scale of the flow and $\beta$ is the local background latitudinal gradient of potential vorticity (comprising, in the simplest context, the relative vorticity and the vorticity due to the planetary rotation). The Rhines scale $L_{R h}$ may be considered as the scale at which zonal motions become important, or, equivalently, the scale at which a background of quasi-two-dimensional turbulent motion begins to project significantly onto the lower frequencies of freely propagating Rossby waves (see e.g. Vallis 2006, for an overview). However, the precise relation between $L_{R h}$ and jet spacing is complicated both by the ambiguity in the choice of $U$, for example, whether it represents an eddy or jet velocity, and by the effect of strong mean flows on the linear Rossby wave propagation. In an evolving flow, it is therefore difficult to predict a priori the spacing of jets that will emerge.

Although forcing mechanisms may vary enormously in different situations, the formation and final structure of jets may be described quite generally in terms of the quasi-horizontal mixing of the background gradient of potential vorticity by eddies. Owing to the constraints of strong stable stratification and rapid rotation, the largescale motions of planetary atmospheres and oceans may, to a first approximation, be characterized by their quasi-two-dimensional motion. Zonal jets arise inevitably when potential vorticity is mixed horizontally over limited latitudinal regions, regardless of the form of the mixing (McIntyre 1982; Dritschel \& McIntyre 2008; Dunkerton \& Scott 2008; McIntyre 2008; Scott 2010). Potential vorticity mixing by planetary-scale Rossby waves in the polar winter stratosphere has already been discussed by McIntyre (1982), who noted the tendency for mixing to weaken potential vorticity gradients in a 'surf zone', while intensifying them on either side, resulting in an intensification of the polar night jet. An analogous effect in the case of vertical mixing by internal waves in a stratified fluid was discussed still earlier by Phillips (1972). In the case of the polar night jet, a large-scale flow is forced by radiative processes, and is eddy intensified into a stronger, sharper jet. However, the material conservation of potential vorticity suggests that any nonlinear eddy motions will suffice to mix the background gradient, which may be considered as 'an unstable equilibrium in the presence of Rossby waves and instabilities' (Dunkerton \& Scott 2008). Thus, while eddies may arise from a variety of sources such as local instability or Rossby wave propagation, their effect on the jet formation may be considered universal in that it involves the mixing of the background potential vorticity. In the cases of the Jovian and oceanic jets, the forcing serves to mix the potential vorticity, but the precise details are of secondary importance.

The so-called potential vorticity staircase describes the limiting case in which the background potential vorticity is mixed perfectly in distinct latitudinal regions, separated by strong gradients at the jet cores. It has been put forward as a model for the Jovian jets (Marcus 1993; Peltier \& Stuhne 2002). In this model, sharp jets correspond to the strong potential vorticity gradients separating mixed zones. It was 
investigated more recently by Dritschel \& McIntyre (2008) and Dunkerton \& Scott (2008), who derived simple expressions for the relation between jet spacing and jet strength, reviewed in $\S 2$ below. In this paper, a second question is addressed, namely, under what conditions, if any, can the staircase limit be achieved in an evolving forced turbulent flow. Or, more generally, what controls the extent of mixing by the turbulent flow. By a series of numerical experiments, described in $\S 4$ below, we demonstrate that the extent of mixing is controlled by a single non-dimensional parameter that may be conveniently expressed as a ratio of two length scales: the Rhines scale $L_{R h}$, and a length scale, introduced originally by Maltrud \& Vallis (1991), that describes the intensity of the forcing relative to the background potential vorticity gradient $L_{\varepsilon}=\left(\varepsilon / \beta^{3}\right)^{1 / 5}$, where $\varepsilon$ is the rate of energy input by the forcing. Essentially, the strength of zonal jets and the steepness of potential vorticity gradients in the jet cores increase as the ratio $L_{R h} / L_{\varepsilon}$ is increased. The staircase limit is approached for $L_{R h} / L_{\varepsilon} \sim O(10)$. For $L_{R h} / L_{\varepsilon} \sim O(1)$, below a value of around 5, on the other hand, the jets and the potential vorticity gradients in the jet cores remain strongly perturbed by the eddy activity of the background turbulent flow.

The dependence of jet strength on a parameter similar to $L_{R h} / L_{\varepsilon}$ has been discussed previously (Vallis 2006; Sukoriansky, Dikovskaya \& Galperin 2007), but largely on phenomenological grounds, and in relation to the halting of the inverse cascade through frictional effects. In the two-dimensional barotropic equations under consideration, with specified $\beta$, total energy, and energy input rate, it is the single nondimensional parameter controlling the system state. However, the relation between this parameter and the organization of potential vorticity into a staircase-like distribution has not previously been considered. Part of the reason for this is that previous numerical experiments have typically not observed flows with strong staircase-like distributions (e.g. Sukoriansky et al. 2007), leading some to question the realizability of such distributions in any physical system. As demonstrated here, the staircase limit is indeed realizable, but only under conditions of exceptionally weak forcing, for which the staircase emerges on correspondingly long time scales. The extremely long integration times required, together with the need for high resolution, may partly explain why such strong staircase-like distributions have not been observed and why the simple relation to $L_{R h} / L_{\varepsilon}$ has not been documented.

Of course, the structure of jets might also depend not just on the total energy and the energy input rate of the forcing, but also on details of the physical mechanism through which the forcing increases the energy of the system. In reality, a quasi-twodimensional atmospheric flow may be forced via a variety of physical mechanisms, including shear instability, both barotropic and baroclinic, convective penetration from an underlying layer, or flow over topography. The common practice in numerical investigations of two-dimensional turbulence is to ignore such distinctions and use a simple band-limited spectral-space forcing as the crudest representation of all these physical processes, the tacit assumption being that the energy input rate is the most important factor controlling the turbulent evolution. Band-limited spectralspace forcing may be the most relevant representation of forcing via shear instability in baroclinic models, where the dominant unstable mode is located at a distinct wavenumber (which in a fully three-dimensional model would be controlled to an extent by the internal deformation radius). To examine the degree to which the forcing mechanism does influence the final jet structure, we consider three qualitatively distinct types of forcing. In addition to the usual forcing of the band-limited spectralspace type, we also consider two types of forcing with a broad wavenumber spectrum, which may be more relevant to the physical process of convective penetration. The 
three different forcing types and their implementation are discussed further in $\S 3.3$ below. Although the form of the forcing affects some details of the resulting jet structure, we emphasize that our main result, that the staircase limit is approached for $L_{R h} / L_{\varepsilon} \sim O(10)$, is found in all cases.

\section{Jet spacing in the staircase limit}

The natural tendency of eddy motions to mix background potential vorticity explains the ubiquity of zonal jets in atmospheres and oceans and their existence in such a variety of physical situations. The evolution of the potential vorticity field towards a staircase-like distribution may be understood intuitively as a result of the tendency of strong potential vorticity gradients to suppress further (latitudinal) mixing, an effect described as Rossby wave elasticity: a perturbation made to a region of strong gradients will be radiated as Rossby waves, rather than result in an irreversible deformation of the gradients themselves. Conversely, regions of weak potential vorticity gradients are highly susceptible to deformation and further mixing. The combined result is a positive feedback whereby mixing is enhanced in regions of weak gradients and suppressed in regions of strong gradients. When waves or eddies are present in the flow, any local reduction or intensification of potential vorticity gradients will be enhanced. If the mixing/steepening processes are allowed to continue unabated (the conditions for this will be discussed in $\S 4$ below) then the potential vorticity structure will evolve into a staircase-like structure, comprising a monotonic piecewise-constant distribution in latitude (regions of zero potential vorticity gradient separated by isolated discontinuities or jumps in latitude). See recent reviews by Baldwin et al. (2007), McIntyre (2008) and Scott (2010) for further discussion.

The jumps of potential vorticity in latitude correspond, through the diagnostic relation between potential vorticity and velocity, to sharply peaked, zonally aligned, eastward jets (see e.g. Dritschel \& McIntyre 2008, figure 7). It is worth noting that the above description explains quite naturally the asymmetry between eastward and westward jets sometimes discussed in the literature. In this description, only the eastward jets are true jets, the westward flow being merely a return flow required by mass conservation. Put another way, the regions of steep potential vorticity gradients naturally define the jet cores. The distinction is important when considering jets as potential barriers to the latitudinal transport of chemical species.

The limit of a zonally symmetric perfect potential vorticity staircase, composed of monotonic piecewise-constant potential vorticity in the latitudinal direction, allows a simple analytic relation to be obtained relating the spacing between the jumps to the strength of the associated jets. Two separate cases were considered recently: single-layer quasi-geostrophic barotropic flow on the sphere (Dunkerton \& Scott 2008); and single-layer quasi-geostrophic equivalent barotropic flow on the $\beta$-plane (Dritschel $\&$ McIntyre 2008). In both cases the half-separation of the jets, $L_{j}$, or the distance between adjacent peak eastward and westward velocity, is given by

$$
L_{j}=\sqrt{3} L_{R h}
$$

where $L_{R h}$ is defined with $U$ denoting the peak eastward velocity. (Note that the prefactors of $\sqrt{2}$ and $\sqrt{6}$ used in Dritschel \& McIntyre 2008 and Dunkerton \& Scott 2008, respectively, result from slight variations in the definition of $L_{R h}$.) In the equivalent barotropic case studied by Dritschel \& McIntyre (2008), the result is exact in the limit of infinite Rossby deformation length, $L_{D}$ (defined in the parent shallow water system as the ratio of gravity wave speed to rotation). In the barotropic case 
studied by Dunkerton \& Scott (2008), the result is asymptotic in latitude, being exact for a staircase profile localized near the equator, but holding to a good approximation in midlatitudes. The result is essentially dependent only on the background angular momentum profile having a quadratic dependence in the latitudinal coordinate (sine of latitude on the sphere).

The result can be extended qualitatively to the case of a smoother potential vorticity distribution (retaining the assumption of zonal symmetry). If, starting from a staircase of a given jet separation, we smooth the jumps in potential vorticity at the jets, we reduce the maximum jet speed while retaining the same jet separation. Conversely, a smooth staircase with the same jet speed would have jets that were spaced farther apart than those of the limiting case described above. The relation between jet spacing and jet speed may therefore be considered as an inequality

$$
L_{j} \geqslant \sqrt{3} L_{R h},
$$

which holds for all zonally symmetric profiles, and with equality for the piecewiseconstant staircase described above.

In the numerical experiments described below it is convenient to define $L_{R h}$ not using the maximum jet speed $U$, but rather using the r.m.s. velocity $U_{r m s}$, related to the total energy of the flow. In doing so, the relation between jet spacing and strength is modified slightly to $L_{j} \geqslant 45^{1 / 4} L_{R h}$, the prefactor here being increased by a factor of $5^{1 / 4}$. (This inequality is valid provided, of course, that $U_{r m s}$ is taken as the r.m.s. of $\bar{u}(y)$, that is, neglecting the energy in the eddy field. In fact, for well-developed jet flows in the barotropic case studied here, nearly all the energy is indeed contained in the zonal mean flow, significant eddy kinetic energy being found only in cases very far from the staircase limit, as demonstrated below.)

Although the perfect staircase limit is convenient analytically and provides a bound on the jet spacing of a flow of given energy, the question remains of when, if at all, such complete potential vorticity homogenization may occur in a turbulent flow. Some authors have suggested that only weak potential vorticity mixing takes place resulting in potential vorticity distributions that are far from the staircase limit. On the other hand, staircase-like potential vorticity distributions have certainly been obtained, even at relatively modest numerical resolution (e.g. Scott \& Polvani 2007, figure 10 therein). In that paper, attention was already drawn to the effect of forcing strength on the potential vorticity structure. Figures 2 and 3 of Scott \& Polvani (2007) compared two cases with identical final energy $\mathscr{E}=\varepsilon / 2 r$ in a forced case with frictional damping at rate $r$, but with values of $\varepsilon$ and $r$ that differed by a factor of ten. In the weakest forcing case the jet structure was markedly more pronounced. The numerical experiments described next will examine this question in more detail, varying the forcing and damping over a wider range to explore more carefully the approach to the staircase limit.

\section{The physical model}

\subsection{Equations of motion}

We consider the quasi-geostrophic approximation to the rotating shallow water equations on the $\beta$-plane, where the Coriolis parameter $f=f_{0}+\beta y$ is linear in $y$ with constant gradient $\beta$. We allow for the input of energy via a prescribed forcing and the dissipation of energy by linear friction at rate $r$. The equations reduce to the material advection of quasi-geostrophic potential vorticity, $q$, with the forcing and dissipation 
represented as source terms $\mathscr{F}$ and $-r \zeta$, breaking exact material conservation:

$$
q_{t}+J(\psi, q)=\mathscr{F}-r \zeta,
$$

where $J$ is the Jacobean determinant and $\zeta=\nabla^{2} \psi$ is the relative vorticity. The advecting flow is determined by the streamfunction $\psi$ which is related to $q$ through

$$
q=\beta y+\nabla^{2} \psi-L_{D}^{-2} \psi,
$$

where $L_{D}=\sqrt{g H} / f_{0}$ is the Rossby deformation length and where $g$ is acceleration due to gravity and $H$ is the mean layer depth. Throughout this paper we consider the limit $L_{D}^{-1} \rightarrow 0$ only, corresponding to the case of purely two-dimensional, barotropic vortex dynamics. The case of non-zero $L_{D}^{-1}$, arguably more relevant to the case in which energy is input via baroclinic instability of a background vertical shear (e.g. Panetta 1993), will be treated in a companion paper.

\subsection{Numerical implementation}

Equation (3.1) is integrated numerically in a doubly periodic domain of length $2 \pi$ in both the $x$ - and $y$-directions. The integration is carried out using an extension of the contour-advective semi-Lagrangian algorithm developed by Dritschel \& Ambaum (1997) that both enables the implementation of arbitrary forcing functions $\mathscr{F}$, and more accurately evolves the energy at large scales. Full details and numerical tests of the extension may be found in Fontane \& Dritschel (2009) and Dritschel \& Fontane (2010); here, for completeness, we summarize the main points.

The new algorithm makes use of a three-way decomposition of the potential vorticity field into: (i) a component $q_{s}$ integrated using a pseudo-spectral method for accurate representation of the large-scale flow; (ii) a component $q_{a}$ integrated using a contour-advective method for accurate representation of the intermediate to small-scale flow; and (iii) a component $q_{d}$ used to represent the non-conservative forcing, and to compensate for any errors involved in the contour representation of a smooth field. The latter may be integrated using either a vortex-in-cell method or a pseudo-spectral method as appropriate for the particular choice of forcing (see $\S 3.3$ below). The three fields are combined at each time step using a spectral low-pass filter applied to $q_{s}$ and its complement applied to $q_{a}$, retaining the large-scale parts of $q_{s}$ and the intermediate and small-scale parts of $q_{a}$; the combination of solutions ensures that each method is used where it is most accurate.

At regular intervals, based on the maximum vorticity of the flow (in practice about every 160 time steps), the contoured field $q_{a}$ is regenerated from the full potential vorticity field, obtained simply by summing the three fields $q_{a}, q_{s}$ and $q_{d}$ on a fine grid. This fine grid, which in the calculations reported here comprises $2048 \times 2048$ grid points over the $2 \pi \times 2 \pi$ domain, thus represents the effective resolution of the integration. Dissipation arising from contour surgery (Dritschel 1989), re-gridding of contours, and gridding of point vortices (when these are used) is effectively confined to scales strictly smaller than the fine grid scale. An important feature is that the contour surgery itself has almost no effect on steep gradients of potential vorticity. The result is an exceptionally weak dissipation compared to other standard methods, as a comparison for the case of isotropic, freely decaying, two-dimensional turbulence recently demonstrated (Dritschel \& Scott 2009). In the integrations reported below, energy dissipation rates due to contour surgery, re-contouring, and gridding are of the order of $10^{-4}$ times smaller than typical dissipation rates due to friction, even in the most weakly damped cases. 
The contoured field $q_{a}$ is advected using a velocity field obtained from the inversion of the combined $q_{a}, q_{s}$ and $q_{d}$ fields interpolated onto a coarse grid comprising $128 \times 128$ grid points. The contour interval used to represent $q_{a}$ is chosen to give 200 contour levels across the domain at $t=0$, i.e. to represent $q$ in the range $[-\beta \pi, \beta \pi]$. This number is sufficient to capture the weak variations of potential vorticity in nearly homogenized regions of the flow at late times.

\subsection{Forcing}

To verify the robustness of our results we consider three qualitatively different types of forcing. The simplest, but arguably least realistic, comprises a narrow-band distribution in spectral space, centred around a specified wavenumber $k_{f}$, and $\delta$-correlated in time. It is popular in studies of the two-dimensional inverse energy cascade because it allows a clear inertial range in spectral space over which the effects of forcing are zero by construction. The function $\mathscr{F}$ in (3.1) is defined in spectral space via its Fourier transform, $\hat{f}_{k}$, which satisfies $\left\langle f_{k} f_{k}^{*}\right\rangle=F(k) / \pi k$ with spectrum $F(k)=\varepsilon$ for $k=|\boldsymbol{k}| \in\left[k_{f}-\delta k, k_{f}+\delta k\right]$ and $F(k)=0$ otherwise, where $\varepsilon$ is the rate of energy input, $\delta k$ is a specified bandwidth, and angle brackets denote an ensemble average. In the numerical implementation, this forcing is added at each time step to the field $q_{d}$, which is periodically recombined with the contour field, as described above.

The above case for which the forcing scale is precisely determined in spectral space may be appropriate as a crude representation of a physical instability that has a well-defined dominant wavelength. This may be contrasted with a physical process such as convective penetration, which may be regarded (at the crudest approximation) as having a well-defined scale, $l_{f}$, in physical space, and a correspondingly broad distribution in spectral space. The second type of forcing used here is designed to represent such a physical process in the case where $l_{f}$ is small. It is implemented numerically through the injection of point vortices of a fixed circulation $\kappa_{v}$, in such a way that the enstrophy input, $\eta$, is constant in time. Because, during the inversion stage, the vorticity of each point vortex is interpolated onto the coarse grid, the length scale of the forcing may therefore be taken as $l_{f}=\delta x$, the coarse grid length scale. The corresponding energy input rate is given approximately by $\eta / k_{f}^{2}$, where $k_{f}=2 \pi / l_{f}$ is the highest wavenumber resolved by the coarse grid, which would be exact if all enstrophy were input at the wavenumber $k_{f}$.

A variety of configurations for the input distribution of point vortices has been considered: they may be input singly, as equal numbers of positive and negative monopoles, randomly distributed in space; as randomly oriented dipole pairs (with zero net circulation); or as quadrupoles (with zero net linear impulse). Likewise, the number of vortices and their circulations must be chosen for a desired enstrophy input rate. All configurations of point vortices give qualitatively similar results, and for brevity we present below only those results obtained with dipole forcing. We note that in this case the orientations of the dipoles are randomized to minimize the systematic input of linear momentum. In the case of quadrupole forcing, for which the linear momentum input is identically zero, each quadrupole tends to split immediately into a dipole pair and so the differences between these two types of forcing are very small. For the dipole distribution, the forcing function $\mathscr{F}$ has energy and enstrophy spectra $F(k)$ and $k^{2} F(k)$ whose forms are shown in figure 1 . Note that the enstrophy is input mainly at the highest resolved wavenumbers, whereas the energy has a much broader distribution in spectral space. The strength of the dipoles is chosen such that the associated maximum absolute vorticity on the grid is $2 \pi \alpha$, for some constant $\alpha$ and from this is calculated the number of dipoles per unit time required to give the correct 


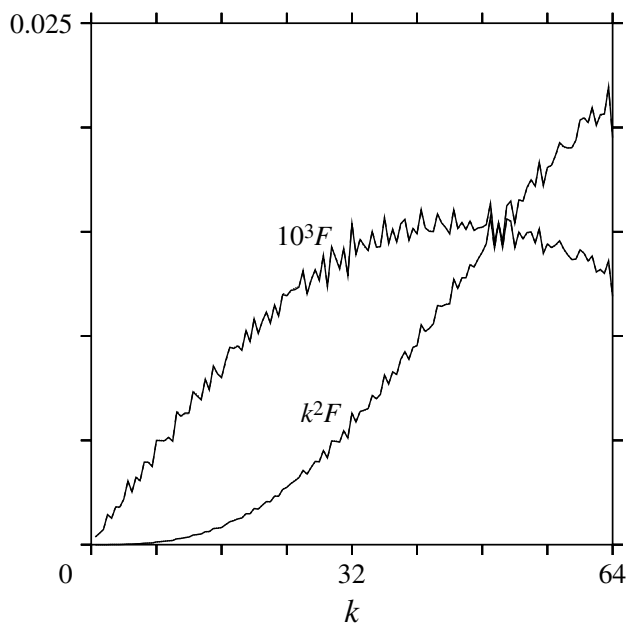

FIGURE 1. Energy and enstrophy spectra of the physical-space forcing using vortex dipoles.

enstrophy input rate. The numerical constant $\alpha$ was set to 0.1 ; tests varying this value between 1 and 0.01 showed no significant effect on the flow evolution.

The third type of forcing considered is a modified version of the physical-space forcing just described. Energy is input in spectral space, with a forcing $\mathscr{F}$ that has the same energy spectrum $F(k)$ as the one shown in figure 1 but with randomized phases of individual Fourier modes. As in the case of narrow-band spectral-space forcing the field $\mathscr{F}$ is again added to the field $q_{d}$.

When $\beta=0$ all of the above types of forcing give a rate of energy input, $\varepsilon_{0}$, into the system that may be specified a priori. When $\beta \neq 0$ the situation is complicated by correlations between the forcing and the zonal mean flows that develop at late times. At early times, say for $t \ll 1 / r$ in the calculations presented below, before strong zonal motions have developed and when the total energy of the flow is small enough that frictional dissipation can be neglected, the total energy $\mathscr{E}$ has been verified to grow as expected like $\varepsilon_{0} t$ for all calculations considered, for some $\varepsilon_{0}$ specified a priori. At later times, however, it was found that the actual energy input rates, $\varepsilon$, as derived from the energy equation, $\dot{\mathscr{E}}=\varepsilon-2 r \mathscr{E}$, where $\mathscr{E}$ is the measured total energy, tend to increase beyond the value $\varepsilon_{0}$. The effect is weak in most cases but is stronger in cases in which the zonal mean flow contains a greater proportion of the total energy, and is more pronounced when the forcing spectrum is broad, presumably because there is greater scope for correlations between the mean flows and the forcing. The effect is strongest in cases of very weak forcing and damping, where the staircase limit is approached, and where the measured energy input rate may be as much as twenty times greater than $\varepsilon_{0}$, depending on the type of forcing used.

Despite possibly significant differences between $\varepsilon_{0}$ and $\varepsilon$, corresponding differences in the key parameters in the system at equilibrium are small. In particular, taking $L_{R h}=\sqrt{U_{r m s} / \beta}=(2 \mathscr{E})^{1 / 4} \beta^{-1 / 2}$, and using $\varepsilon=2 r \mathscr{E}$ at equilibrium, the key nondimensional parameter $L_{R h} / L_{\varepsilon}$ depends on $\varepsilon$ as

$$
L_{R h} / L_{\varepsilon}=\beta^{1 / 10} r^{-1 / 4} \varepsilon^{1 / 20},
$$

the $1 / 20$ th power giving a very weak dependence on the actual value of energy input rate $\varepsilon$ : a measured energy input rate $\varepsilon$ exceeding the nominal value $\varepsilon_{0}$ by a factor 


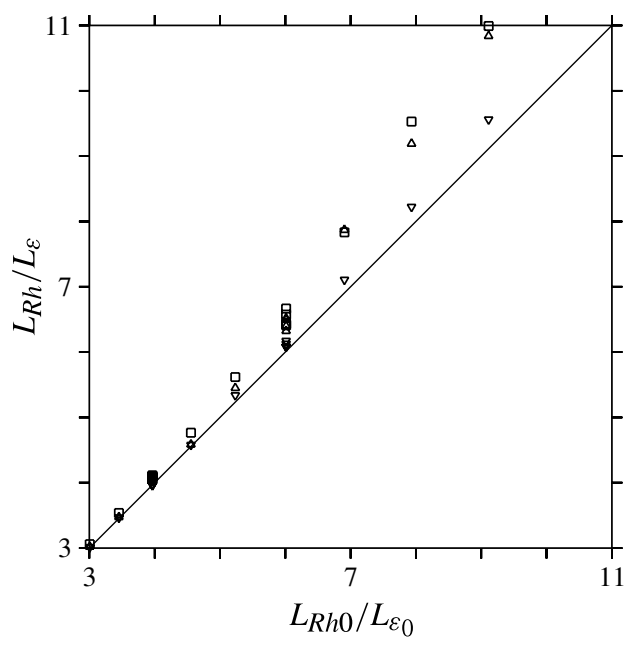

FIGURE 2. Actual $L_{R h} / L_{\varepsilon}$ relative to nominal $L_{R h 0} / L_{\varepsilon 0}$, for $r=1 \times 10^{-4}$ to $r=256 \times 10^{-4}$ and all forcing types: physical-space forcing (triangles), narrow-band spectral-space forcing (inverted triangles), broad-band spectral-space forcing (squares). Multiple symbols for single values of $L_{R h 0} / L_{\varepsilon 0}$ denote multiple realizations of the random forcing.

of twenty, say, will only increase $L_{R h} / L_{\varepsilon}$ by $\sim 16 \%$. To be definite in the analysis of the simulations presented below, however, the energy input rate into the system once a statistical equilibrium has been reached is always calculated a posteriori from the energy equation $\varepsilon=2 r \mathscr{E}$.

\subsection{Physical parameters}

Equation (3.1) is non-dimensionalized such that the domain length $L=2 \pi$, and we consider here only the case $L_{D}^{-1}=0$. A natural time scale may be defined in terms of the Rossby wave frequency for flows with a nominal characteristic length scale $L_{R h 0}$, as $T_{\beta}=2 \pi / \beta L_{R h 0}$. Here, $L_{R h 0}$ is defined as the Rhines scale (defined as $L_{R h}=\sqrt{U_{r m s} / \beta}$ ) that would be obtained with a specified frictional rate $r$ if the energy input rate $\varepsilon$ were to remain equal to the nominal value $\varepsilon_{0}$ for the duration of the integration. Thus $L_{R h 0}=\left(\varepsilon_{0} / r\right)^{1 / 4} \beta^{-1 / 2}$, and here it is fixed to a value of $L_{R h 0}=1 / 8$, which was found to yield a reasonable number of jets across the domain in the integrations conducted. Setting $\beta=16 \pi$ then gives a convenient time scale of $T_{\beta}=1$, as well as an $O(1)$ value of $U_{0}=\sqrt{\varepsilon_{0} / r}=\pi / 4$.

The choice $L_{R h 0}=1 / 8$ constrains the possible values of $\varepsilon_{0}$ and $r$, through the relation $\varepsilon_{0} / r=\beta^{2} L_{R h 0}^{4}$. Keeping the ratio $\varepsilon_{0} / r$ fixed, $\varepsilon_{0}$ and $r$ are varied across a range of values to achieve $L_{R h 0} / L_{\varepsilon 0}$ approximately in the range $[3,10]$, where $L_{\varepsilon 0}=\left(\varepsilon_{0} / \beta^{3}\right)^{1 / 5}$. Specifically $r$ is varied from 0.0256 , for which $L_{R h 0} / L_{\varepsilon 0} \approx 3.0$ to 0.0001 for which $L_{R h 0} / L_{\varepsilon 0} \approx 9.11$. As described in $\S 3.3$, the actual values of $\varepsilon, L_{R h}$, and $L_{\varepsilon}$ are always calculated a posteriori at equilibrium from the measured total energy via $\varepsilon=2 r \mathscr{E}$, and typically exceed the nominal values. For completeness, in figure 2 we show the dependence of the measured values of $L_{R h} / L_{\varepsilon}$ against the nominal values for all simulations conducted: actual values exceed the nominal ones by up to $\sim 18 \%$ for the weakest forcing cases and by a smaller amount at stronger forcing. 
(a)

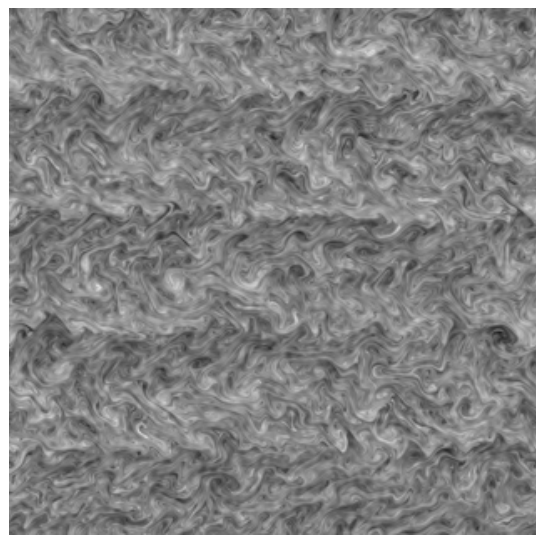

(c)

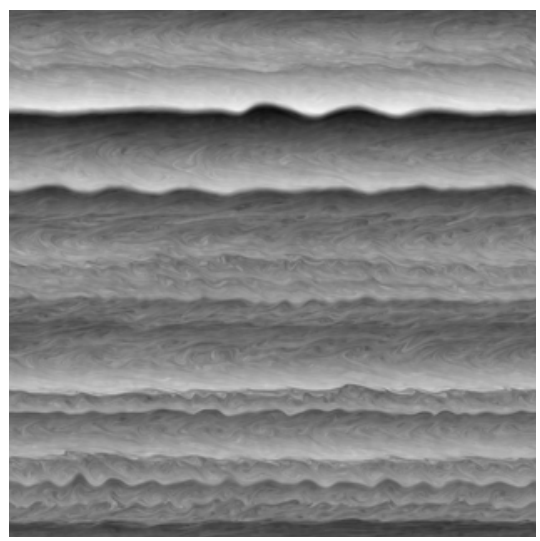

(b)

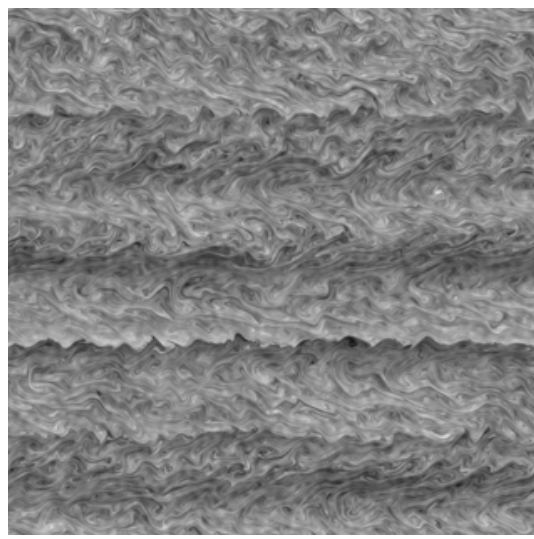

$(d)$

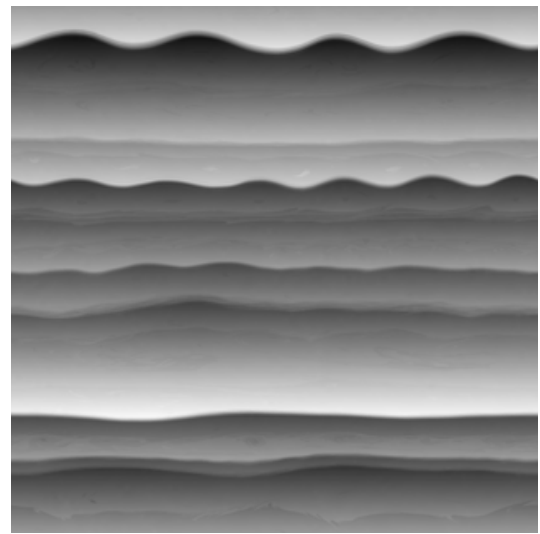

FIgURE 3. Potential vorticity anomaly $q-\beta y$ at $t=10 / r$ for $r=(256,64,8,1) \times 10^{-4}$ $(a-d)$ and physical-space forcing; $L_{R h} / L_{\varepsilon}=3.0,4.0,6.5,10.8$, respectively.

The energy of the flow equilibrates to the final value on a time scale of $1 / r$. To ensure that the flow reaches a stationary state with respect to the formation of zonal jets, we integrate (3.1) for a total time of $10 / r$. The stationarity of the flow by this time is verified in the numerical experiments below.

\section{Realizability of the staircase}

\subsection{Physical-space forcing}

The results from the numerical experiments are presented next. We focus mainly on the case of physical-space forcing by point vortex dipoles, as described in $\S 3.3$ above. The results are compared with the case of spectral-space forcing in $\S 4.3$.

Figure 3 shows the potential vorticity anomaly $q(x, y, t)-\beta y$ at the final integration time $t=10 / r$ for four cases, $r=0.0256,0.0064,0.0008,0.0001$ (with the nominal $\varepsilon_{0}$ given through the relation $\left.L_{R h 0}=\left(\varepsilon_{0} / r\right)^{-1 / 4} \beta^{-1 / 2}=1 / 8\right)$. The corresponding values of $L_{R h} / L_{\varepsilon}$, computed from the measured $\mathscr{E}$, are $3.0,4.0,6.5$ and 10.8 , respectively. It is immediately clear that the structure of the turbulent flow arising from the forcing is changing systematically with $L_{R h} / L_{\varepsilon}$, transitioning from almost homogeneous eddydominated turbulence at small values to a highly inhomogeneous field dominated by distinct zonal bands at large ones. These bands comprise regions over which the 
(a)

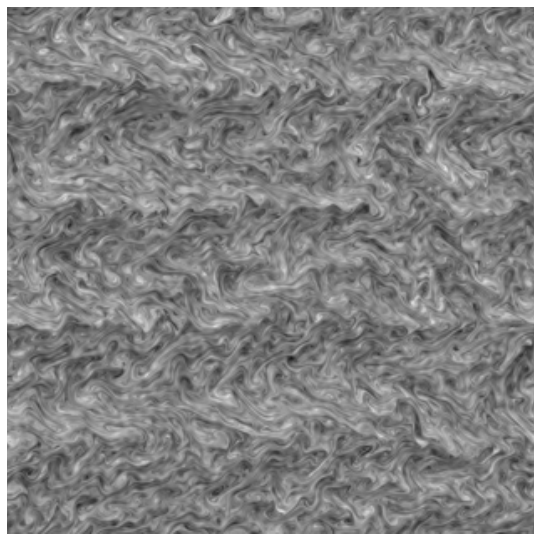

(a)

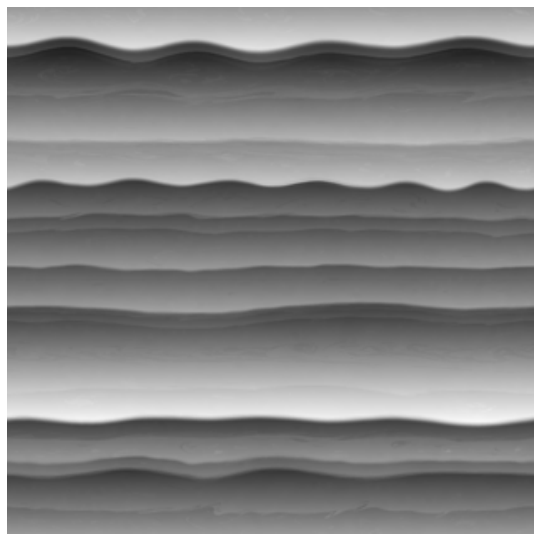

FIGURE 4. Potential vorticity anomaly $q-\beta y$ at $t=4 / r$ for the cases $(a) r=256 \times 10^{-4}$ $\left(L_{R h} / L_{\varepsilon}=3.0\right)$ and $(b) r=1 \times 10^{-4}\left(L_{R h} / L_{\varepsilon}=10.8\right)$.

potential vorticity is almost perfectly mixed in $y$ (so that the anomaly $q-\beta y$ has a linear gradient) separated by sharp jumps, or near discontinuities in $y$. As we show below the structure of the potential vorticity field for $L_{R h} / L_{\varepsilon}=10.8$ is close to the staircase distribution described in $\S 2$, with the sharp jumps corresponding to strong zonal jets.

To illustrate that the integrations have reached statistical equilibrium, we show in figure 4 the potential vorticity anomaly (the same fields) from the extreme cases $r=0.0256$ and $r=0.0001$ at the earlier time $t=4 / r$. The fields are not only qualitatively similar to their counterparts at $t=10 / r$, but are also similar in many details. For example, the 'ghost jets' just visible in the large- $r$ case are in approximately the same positions at $t=4 / r$ and $t=10 / r$; images of the fields between these times verify that the jets are quasi-stationary over this time interval. Similarly, the locations of the strong potential vorticity jumps and associated jets in the small-r case are almost stationary, in some cases somewhat surprisingly: the two lowermost neighbouring jets in the figure, for example, persist throughout the entire time period and show no sign of merging into a single large jet. In fact, such jet merger would be difficult energetically, since two small jumps in potential vorticity coalescing into a single larger jump would involve a local increase in the energy of the jet. The increase could be offset in two ways: either (i) by a coalescence into a single jump that is smoothed out in $y$, across which the potential vorticity gradient is smaller; or (ii) by a coalescence into a single jump on which the energy is reduced by the growth of substantial wave motions. In the case of (i), a reduction in the potential vorticity gradient would correspond to a down-gradient potential vorticity flux due to either diffusion or through advective potential vorticity mixing. Here, diffusion across potential vorticity contours is effectively zero, while in the weakly forced case it appears that the eddy field is too weak to mediate the required reduction in potential vorticity gradient. The strength of the eddy field is discussed in more detail below. In the case of (ii), the energy reduction due to waves on the jet, that have at most $O(1)$ slopes, is evidently insufficient to compensate for the energy increase due to jet merger.

The zonal mean structure is shown in figure 5 for the two extreme cases $L_{R h} / L_{\varepsilon}=3.0(r=0.0256)$ and $L_{R h} / L_{\varepsilon}=10.8(r=0.0001)$, with the zonal-mean zonal 
(a)

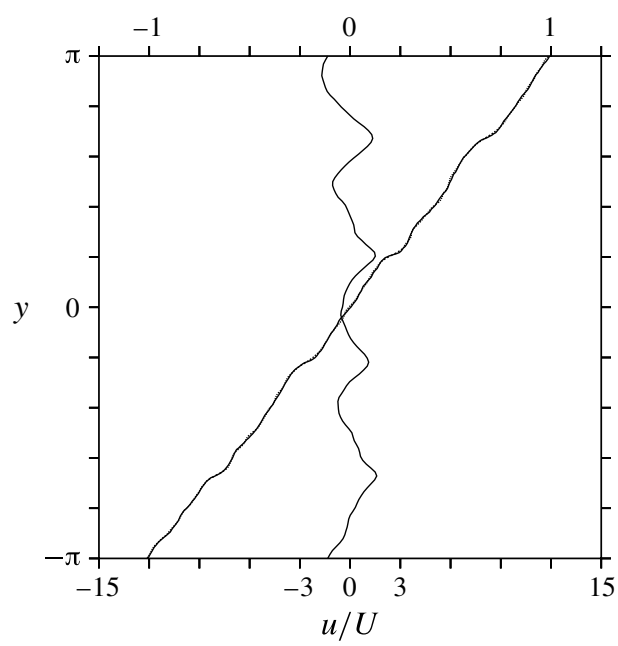

(b)

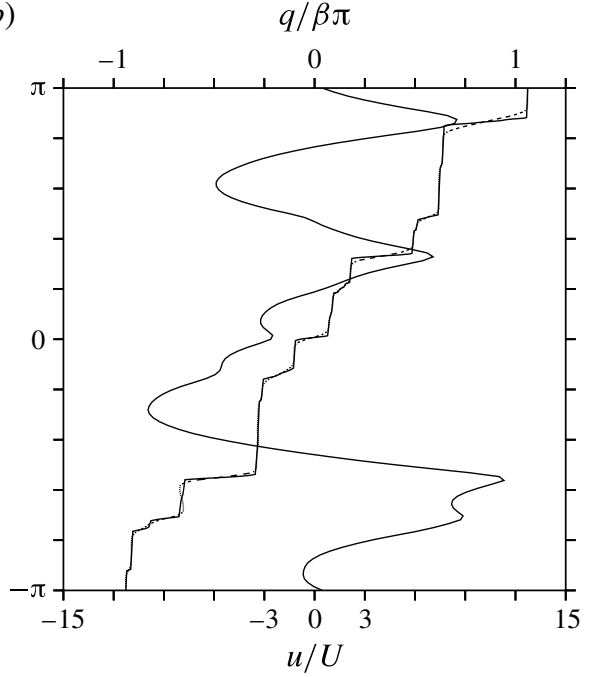

FIGURE 5. $\bar{u}(y)$ (solid line), $\bar{q}(y)$ (dotted line) and $q\left(y_{e}\right)$ (solid line) at $t=10 / r$ for the cases (a) $r=256 \times 10^{-4}\left(L_{R h} / L_{\varepsilon}=3.0\right)$ and $(b) r=1 \times 10^{-4}\left(L_{R h} / L_{\varepsilon}=10.8\right.$, corresponding to figure $3(a, d)$. Velocities are scaled by $U=\sqrt{\varepsilon_{0} / r}$.

velocity $\bar{u}(y)$ as the solid line, and the zonal-mean potential vorticity $\bar{q}(y)$ as the dotted line. For the case $L_{R h} / L_{\varepsilon}=3.0$ four weak jets are visible corresponding to four main regions of enhanced potential vorticity gradients; maximum gradients here are larger than $\beta$ but not dramatically so. Similarly, the regions between the jets have potential vorticity gradients smaller than $\beta$, but do not show complete mixing. In contrast, for the case $L_{R h} / L_{\varepsilon}=10.8$, potential vorticity is almost completely mixed in regions separated by abrupt jumps with gradients much greater than $\beta$, these corresponding to strong jets of magnitude proportional to the size of the jump. We note that details of these profiles such as the position and strength of individual jets are not unique, but vary for different realizations of the random forcing; results of other realizations are summarized in $\S 4.4$ below.

The zonal averaging of $q(x, y, t)$ necessarily leads to a reduction in the across-jet gradient for jets on which significant wave motions exist, for example the strong jet near the top of the domain at $y=\pi$. To obtain a clearer picture of the jet structure, an equivalent latitude measure may be used, in which the area occupied by potential vorticity values greater than a threshold value $q$ is used to define an equivalent latitude $y_{e}(q)$. Its inverse $q\left(y_{e}\right)$ is well-defined since $y_{e}$ is a monotonic function of $q$ by construction. A further refinement is used here, which considers only the potential vorticity associated with open contours, that is, contours that span, or wrap the domain in the $x$-direction, a procedure introduced in Dritschel \& Scott (2011) to limit the effect of strong coherent vortices residing between jets. The refinement is most relevant to cases (not considered here) of finite deformation radius. In the present case, the difference between $y_{e}$ defined by the full potential vorticity field and $y_{e}$ defined by wrapping contours only is in fact very small; we retain the latter calculation for consistency with a companion paper considering the case $L_{D}^{-1}>0$.

The equivalent-latitude-based measure $q\left(y_{e}\right)$ is shown in figure 5 by the solid lines. For the case of $L_{R h} / L_{\varepsilon}=3.0$ there is no appreciable difference between it and the zonal mean $\bar{q}(y)$. For the case $L_{R h} / L_{\varepsilon}=10.8$, the quantity $q\left(y_{e}\right)$ in places better 
(a)

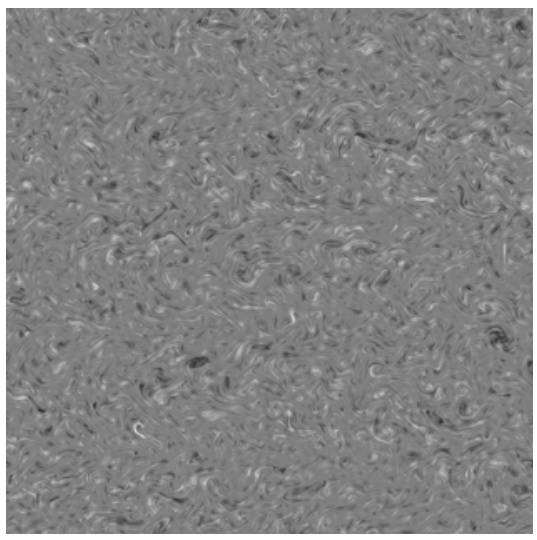

(b)

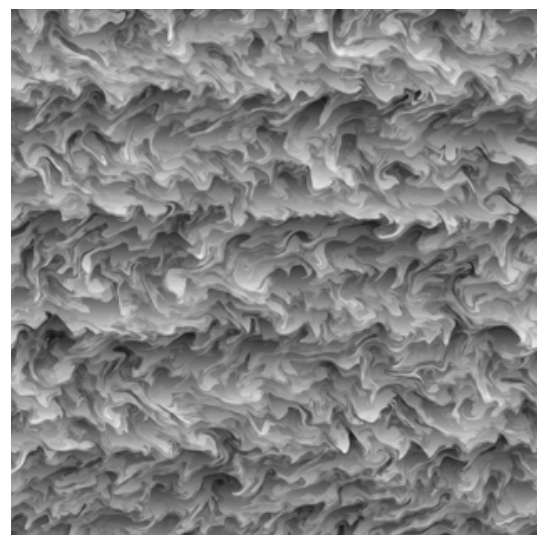

FIGURE 6. Potential vorticity anomaly $q-\beta y$ associated with $(a)$ the eddy residual and $(b)$ jets at $t=10 / r$ for the case $r=256 \times 10^{-4}\left(L_{R h} / L_{\varepsilon}=3.0\right)$, corresponding to figure $3(a)$.

illustrates the sharpness of the potential vorticity jumps associated with the jets, for example, at the jet just below $y=\pi$, or around $y=0, \pi / 3,-\pi / 2$, which exhibit near discontinuities in $q\left(y_{e}\right)$. For nearly straight jets the difference between $\bar{q}(y)$ and $q\left(y_{e}\right)$ is of course minimal.

As a final remark, it is worth noting the irregularity of the jet structure in the case $L_{R h} / L_{\varepsilon}=10.8$, which may be characterized by three strong jets with weaker jets existing on the flanks of the stronger ones. Their precise locations determine their directions: the jet near $y=0$ has a local maximum in velocity that is, however, negative in the rest frame. In contrast, the jet near $y=-3 \pi / 4$ is a positive local maximum near the global maximum just to the north. The velocity profile between the two maxima has an approximately parabolic profile in $y$ (since the potential vorticity is nearly constant) and the double peak structure is not dissimilar to the structure of the super-rotating equatorial jet on Jupiter.

\subsection{Eddy-jet decomposition}

To examine in more detail the structure of the jets and that of the turbulent background, we make a simple decomposition of the potential vorticity field into jet and eddy components. Again, we start by considering the component of the potential vorticity field $q(x, y, t)$ consisting of contours that wrap the domain in the $x$-direction. We then decompose the full $q(x, y, t)$ into a component $q_{j e t}$ associated with these contours, and into a component $q_{e d d y}$ associated with the residual. The decomposition is shown in figure 6 for the case $L_{R h} / L_{\varepsilon}=3.0(r=0.0256)$ and in figure 7 for the case $L_{R h} / L_{\varepsilon}=10.8(r=0.0001)$. In both cases it can be seen that the decomposition has effectively removed all zonally symmetric contributions from the field $q_{\text {eddy }}$ (panels $a$ ). In the case $L_{R h} / L_{\varepsilon}=3.0$ the $q_{e d d y}$ field appears broadly homogeneous. Here, the jets are too weak to organize the turbulent eddy field in a systematic way. In contrast, for $L_{R h} / L_{\varepsilon}=10.8$ the eddies can be seen to be organized by the jets, for example the row of six cyclonic (white) eddies just north of the jet at $y=\pi / 3$ (and a corresponding row of anticyclonic eddies to the south). These eddies are a manifestation of critical layer mixing by the wavenumber-six wave existing on the jet.

Energy spectra for the full, jet and eddy potential vorticity fields are shown in figure 8. For the case $L_{R h} / L_{\varepsilon}=3.0$, the spectrum is dominated by the jet 
(a)

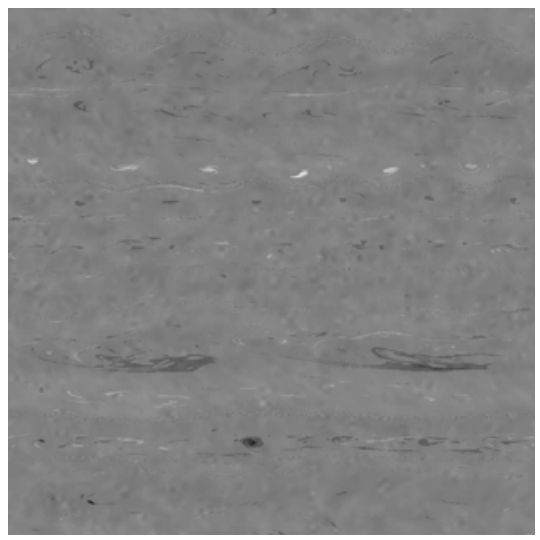

(b)

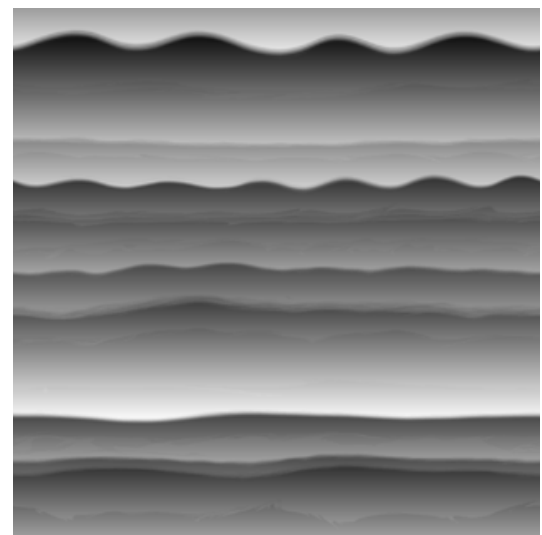

FIGURE 7. Potential vorticity anomaly $q-\beta y$ associated with $(a)$ the eddy residual and $(b)$ jets at $t=10 / r$ for the case $r=1 \times 10^{-4}\left(L_{R h} / L_{\varepsilon}=10.8\right)$, corresponding to figure $3(d)$.
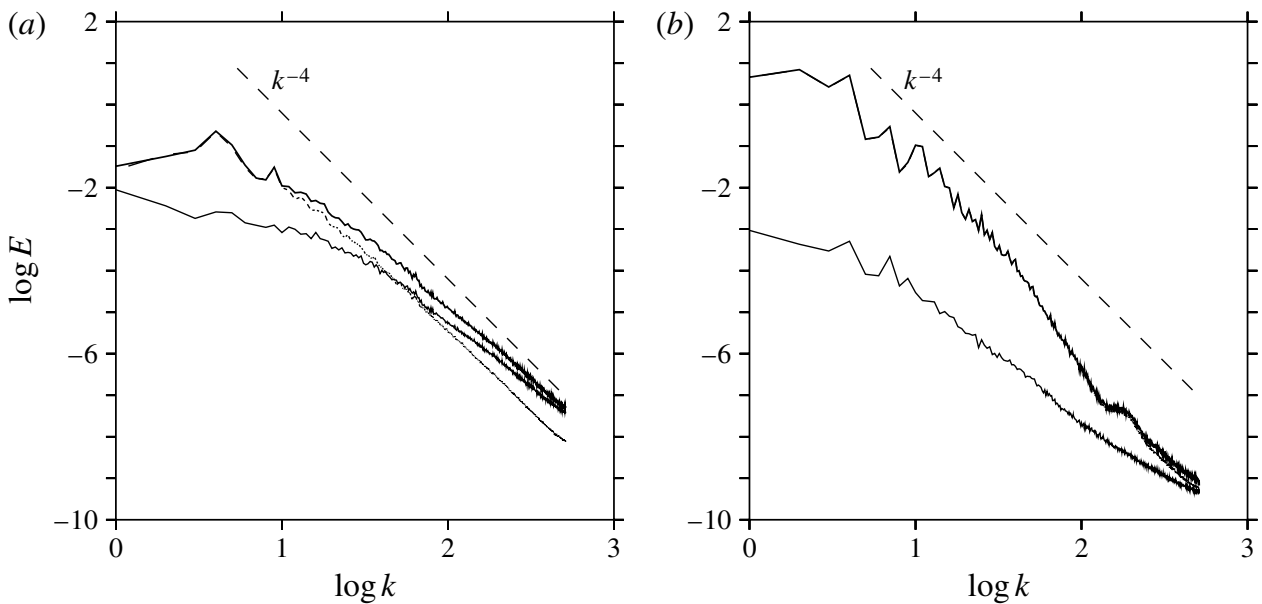

FIGURE 8. Energy spectra computed from the full (bold), jet (dotted), and eddy (thin solid) vorticity fields for the $(a)$ cases $r=256 \times 10^{-4}\left(L_{R h} / L_{\varepsilon}=3.0\right)$ and $(b) r=1 \times 10^{-4}$ $\left(L_{R h} / L_{\varepsilon}=10.8\right)$, corresponding to figure $3(a, d)$.

component $q_{j e t}$ at the largest scales or smallest wavenumbers $k$ only; at wavenumbers greater than about $k=10$ the fraction of the total energy contained in $q_{\text {jet }}$ begins to decrease and the fraction contained in $q_{e d d y}$ begins to increase. The cross-over occurs around $k=60$, beyond which most of the energy is contained in $q_{\text {eddy. }}$. It is noteworthy that, despite the fact that the jets in this case are so indistinct, most of the total energy $(81.8 \%)$ is nevertheless contained in the field $q_{j e t}$. The overall shape of the full energy spectrum is approximately $k^{-3}$. The shape of the spectrum associated with $q_{j e t}$ transitions from approximately $k^{-3}$ at low $k$ to $k^{-4}$ at high $k$ (above approximately $k=30)$.

For the case $L_{R h} / L_{\varepsilon}=10.8$, in contrast, the full energy spectrum is dominated by the contribution from $q_{\text {jet }}$ across all wavenumbers, all the way down to the 
(a)

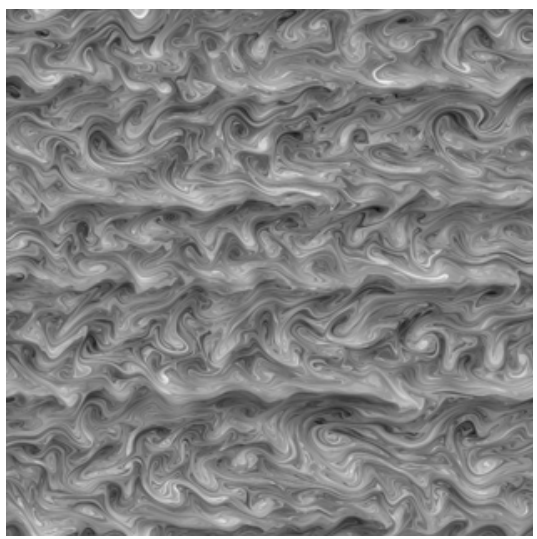

(b)

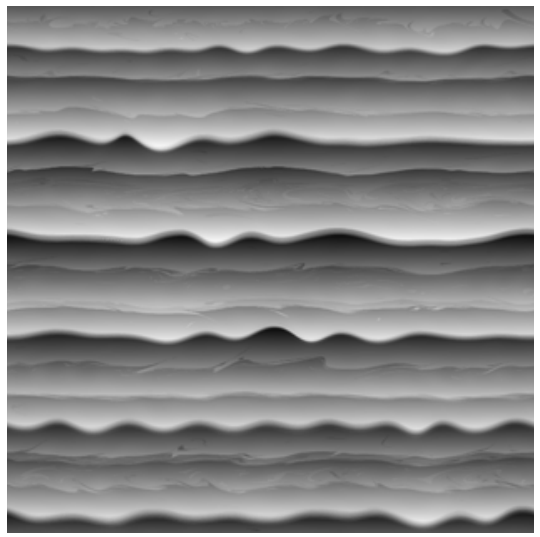

FIGURE 9. Potential vorticity anomaly $q-\beta y$ at $t=10 / r$ for the cases $(a) r=256 \times 10^{-4}$ $\left(L_{R h} / L_{\varepsilon}=3.0\right)$ and $(b) r=1 \times 10^{-4}\left(L_{R h} / L_{\varepsilon}=9.6\right)$ and narrow-band spectral-space forcing.

smallest scales $\left(q_{\text {jet }}\right.$ contains $98.7 \%$ of the energy). At large scales, $E(k) \sim k^{-4}$, which is expected for a staircase in which the structure is dominated by a series of discontinuities in $y$, similar to the spectrum proposed by Saffman (1971) for the case of two-dimensional isotropic turbulence. Discontinuities in a one-dimensional field $q(y)$ imply a Fourier series whose coefficients fall off as $k^{-1}$, giving an enstrophy spectrum of $k^{-2}$ and an energy spectrum of $k^{-4}$. The staircase structure thus has a shallower spectrum than the $k^{-5}$ shape suggested by other authors (e.g. Huang, Galperin \& Sukoriansky 2001).

\subsection{Forcing comparison}

The basic dependence of jet structure on $L_{R h} / L_{\varepsilon}$ described above was found regardless of the particular choice of the type of forcing (out of the three types described in $\S 3.3$ ). Both narrow- and broad-band (random phased) spectral-space forcing produced the same increase in jet strength and approach to the staircase limit as the forcing and damping were reduced, with distinct staircasing appearing for $L_{R h} / L_{\varepsilon}$ above around 6 (quantified further in $\$ 4.4$ below). For comparison, figure 9 shows the full potential vorticity anomaly, $q(x, y, t)-\beta y$, at $t=10 / r$ for the two cases $r=0.0256$ and $r=0.0001$ for narrow-band spectral-space forcing. Measured values of $L_{R h} / L_{\varepsilon}$ for these cases are 3.0 and 9.6, respectively. A similar pattern to that shown in figure 3 can be seen. In the case $r=0.0256$, where $L_{R h} / L_{\varepsilon}=3.0$ for both forcing types, eddies are on the whole larger than those in figure $3(a)$ as a result of the larger-scale forcing $\left(k_{f}=16\right)$; the corresponding case of broad-band forcing with random phases but identical forcing spectrum to that shown in figure 1 has a $q(x, y, t)-\beta y$ field (not shown) almost indistinguishable in structure from that shown in figure $3(a)$.

The most significant difference between the narrow-band and physical-space forcing is found for the case $r=0.0001$ in the regularity of the jet spacing (compare figures $3 b$ and $9 b$ ). The values of $L_{R h} / L_{\varepsilon}$ are comparable, 9.6 and 10.8 respectively, and extremely large and small gradients of potential vorticity are found in each case. However, the narrow-band forcing results in jets that are much more regular in both spacing and intensity than those obtained with physical-space forcing. The zonal-mean zonal velocity profile, shown in figure 10, comprises six main jets all of comparable strength. The regularity of these jets appears to arise from the lack of projection of 
(a)

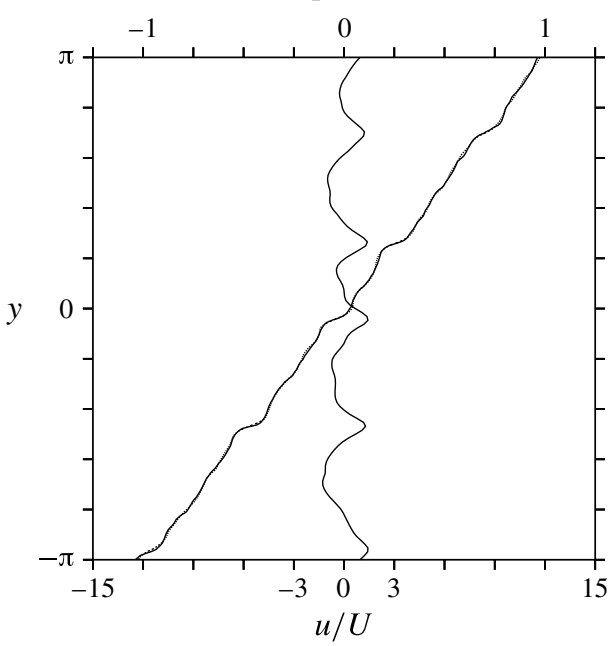

(b)

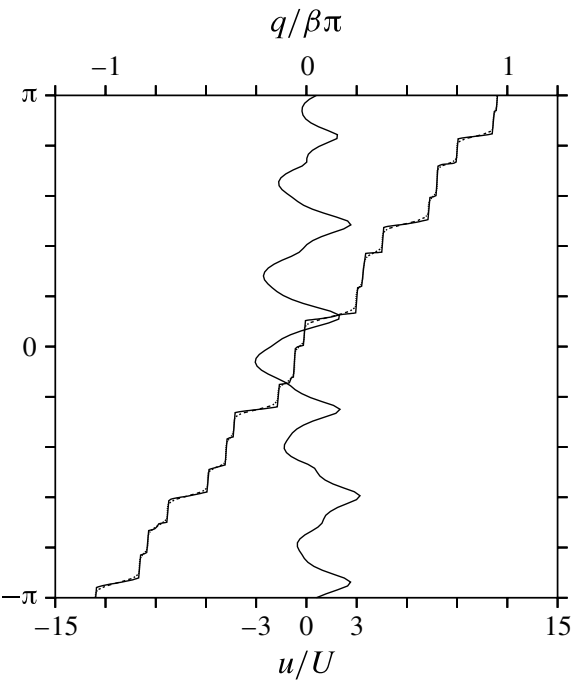

FIGURE 10. $\bar{u}(y)$ (solid line), $\bar{q}(y)$ (dotted line) and $q\left(y_{e}\right)$ (solid line) at $t=10 / r$ for the cases $(a) r=256 \times 10^{-4}\left(L_{R h} / L_{\varepsilon}=3.0\right)$ and $(b) r=1 \times 10^{-4}\left(L_{R h} / L_{\varepsilon}=9.6\right)$ corresponding to figure $9(a, b)$.

the narrow-band forcing spectrum onto the large-scale motions. In the physical-space forcing case, the substantial power at the jet scales induces a randomness to the strength and separation of jets, mixing being more intense between some jets than others. In the narrow-band case, in contrast, jets emerge essentially subjected to similar local forcing and grow until they begin to be influenced by neighbouring jets. This argument is supported by the results of the broad-band spectral-space forcing cases, which showed similar jet irregularity to the physical-space forcing cases, suggesting that it is the spectral distribution rather than the phasing that plays the more important role in determining the regularity.

The graphs of $\bar{q}(y)$ and $q\left(y_{e}\right)$ have an interesting structure for the case $L_{R h} / L_{\varepsilon}=9.6$. The six jets are associated with six principle jumps in $q\left(y_{e}\right)$. Between each of these, however, the potential vorticity is not mixed uniformly but has an additional substaircase structure comprising two smaller jumps. The smaller jumps are of insufficient strength to induce local maxima in $\bar{u}(y)$, but lead to smaller departures from a simple parabolic interjet flow. The sub-staircase structure may arise as a result of critical layer mixing on the flanks of the main jets, induced by waves with $O(1)$ slopes existing on the main potential vorticity jumps. However, it should also be noted that the separation between sub-jets corresponds closely to the length scale of the forcing, which is here centred on wavenumber $k_{f}=16$, and so their presence may also reflect direct mixing by the forcing, organized by the main jets.

The energy spectra for the full, eddy and jet fields for the case of narrow-band forcing, shown in figure 11, are similar to those for the case of physical-space forcing. There are some differences in the spectra at high $k$ in the case $L_{R h} / L_{\varepsilon}=9.6$ due to the different spectrum of the forcing, but again there is a short $k^{-4}$ range for $k$ below around $k_{f}$. Here, the local peaks in $E(k)$ reflect the presence of higher harmonics of the main peak at wavenumber six, associated with the regular six-jet structure seen in the zonal mean fields. 

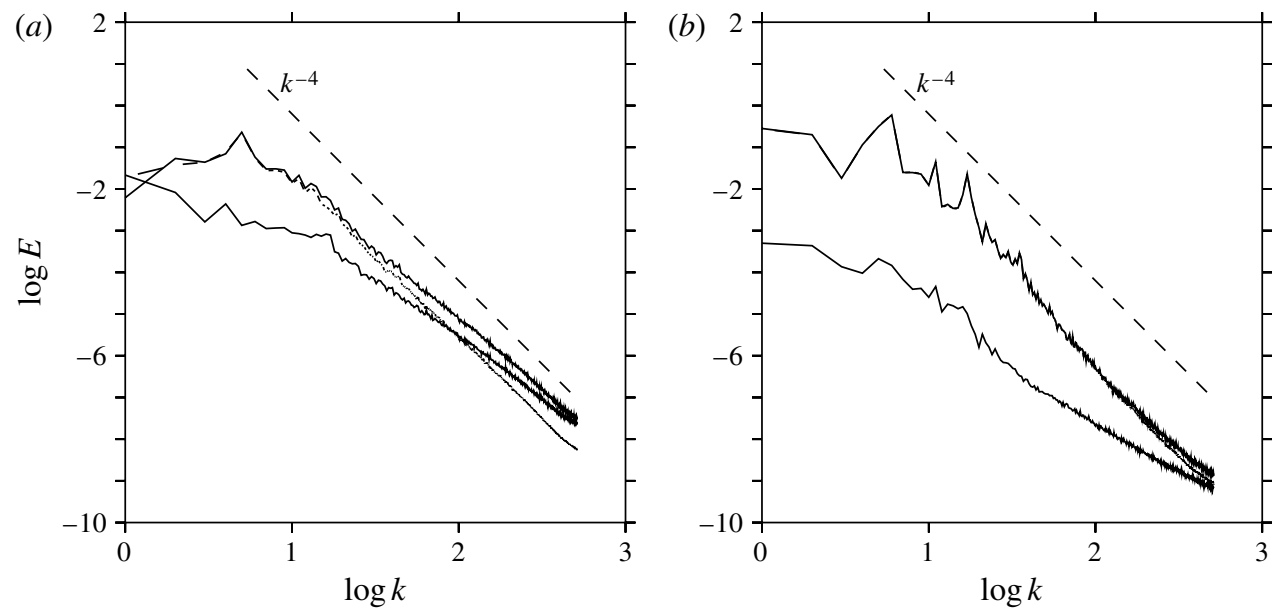

FIGURE 11. Energy spectra computed from the full (bold line), jet (dotted line), and eddy (thin solid line) vorticity fields for the cases $(a) r=256 \times 10^{-4}\left(L_{R h} / L_{\varepsilon}=3.0\right)$ and $(b)$ $r=1 \times 10^{-4}\left(L_{R h} / L_{\varepsilon}=9.6\right)$, corresponding to figure $9(a, b)$.

\subsection{Quantifying the staircase limit}

Finally, we summarize across all calculations the degree to which the staircase limit is approached, and how the approach depends on the parameter $L_{R h} / L_{\varepsilon}$. To this end we describe an objective measure of how close a given $q\left(y_{e}\right)$ profile is to the staircase limit. A simple way in which this may be done is by defining a notional staircase for each profile. For a given $q\left(y_{e}\right)$, we consider the $N$ distinct ranges of $q$ values over which the gradient $\mathrm{d} q / \mathrm{d} y_{e}$ exceeds a critical value, say $3 \beta$. We define the location of a notional stair step $y_{s_{i}}(i=1, \ldots, N)$ as the location corresponding to the midpoint of $q$ in each range. The notional staircase is then constructed by connecting the steps into a monotonic, piecewise-constant profile, $q_{s}$, each step being extended in $q$ between ranges by an amount such that the areas under the staircase and original profile are the same. An example of the construction for the strong jet case of figure $5(b)$, $L_{R h} / L_{\varepsilon}=10.8$, is shown in figure 12 .

Having constructed the notional staircase, $q_{s}$, for each profile, it remains to define a measure of how close the profile is to its staircase. Many measures are possible. Here we consider two, both based on the area difference between the original profile and its staircase. Specifically we define

$$
I_{1}=\int_{-\pi}^{\pi}\left|q-q_{s}\right| \mathrm{d} y_{e}, \quad I_{2}=\int_{-\pi}^{\pi}\left|q_{s}-\beta y\right| \mathrm{d} y_{e}, \quad I_{3}=\int_{-\pi}^{\pi}|q-\beta y| \mathrm{d} y_{e},
$$

as the area between the original profile $q$ and its staircase $q_{s}\left(I_{1}\right)$; the area between the staircase and the line $\beta y\left(I_{2}\right)$; and the area between the original profile and the line $\beta y\left(I_{3}\right)$. Values of $I_{1}, I_{2}$ and $I_{3}$ are time averaged over the last half of each integration (from $t=5 / r$ to $t=10 / r$ ) to reduce the variance of the values obtained. Two simple measures of how well $q_{s}$ approximates $q$ are then given by

$$
1-I_{1} / I_{2} \text { and } I_{3} / I_{2} \text {, }
$$

which vary between zero and one: both are zero for the case $q=\beta y$, and both are one for the staircase limit $q=q_{s}$. 


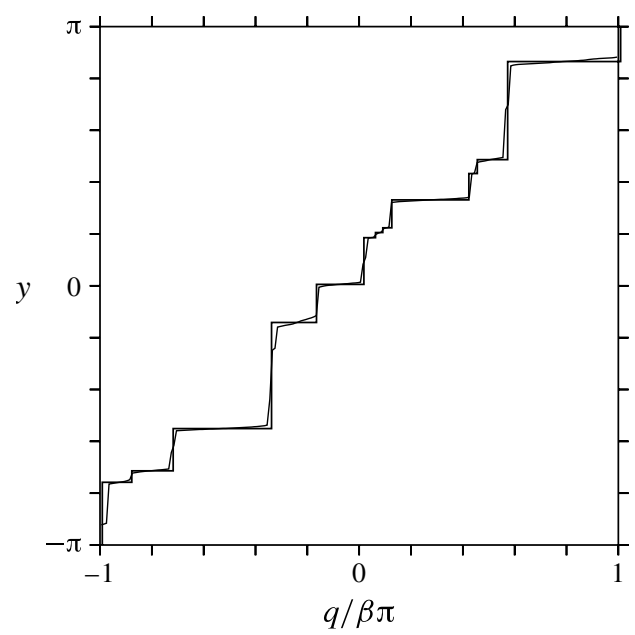

FIGURE 12. The profile $q\left(y_{e}\right)$ from the case of physical-space forcing and $r=0.0001$ $\left(L_{R h} / L_{\varepsilon}=10.8\right.$, see figure $\left.5 b\right)$ together with a notional staircase constructed as described in the text.

(a)

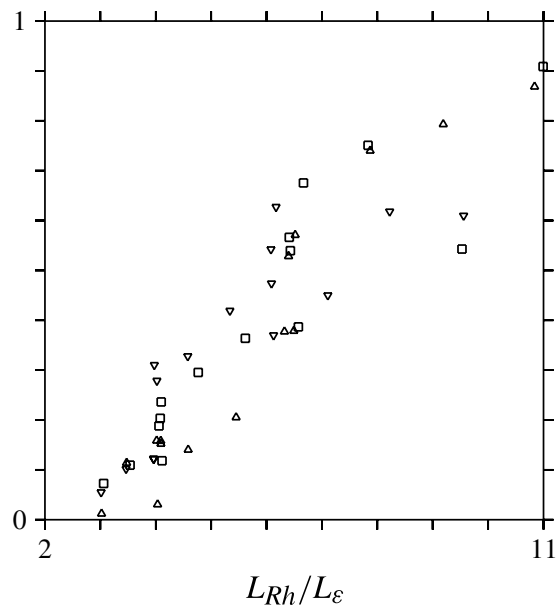

(b)

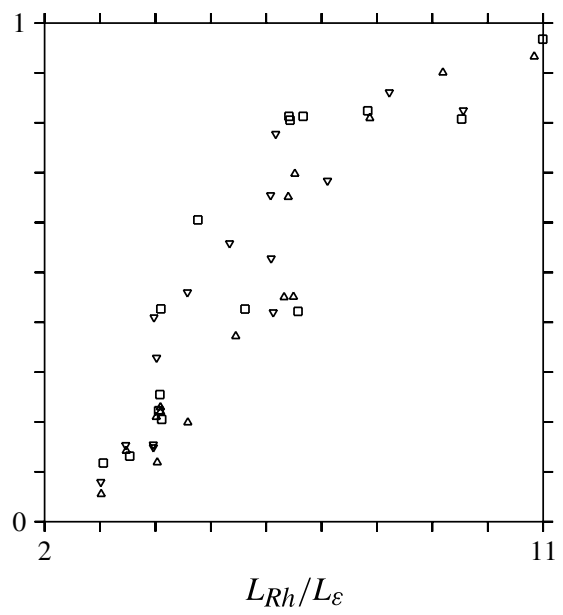

FIGURE 13. Closeness of fit to the staircase limit, $1-I_{1} / I_{2}(a)$ and $I_{3} / I_{2}(b)$ as a function of $L_{R h} / L_{\varepsilon}$ (see text for details); physical-space forcing (triangles), narrow-band spectral -space forcing (inverted triangles), broad-band spectral-space forcing (squares). Multiple symbols for single values of $L_{R h} / L_{\varepsilon}$ denote multiple realizations of the random forcing.

The results for all the calculations are shown in figure 13, where the two measures in (4.2) are plotted against the parameter $L_{R h} / L_{\varepsilon}$. Both measures show qualitatively the same dependence, namely a steady increase towards the staircase limit with increasing $L_{R h} / L_{\varepsilon}$. It appears that the second measure $I_{3} / I_{2}$ is a more robust indicator of the staircase limit at values of $L_{R h} / L_{\varepsilon}$ above $\sim 8$, where all cases develop strong staircasing. In the case of the physical-space forcing (triangles), there is some evidence in both measures of a more abrupt transition from low values (far from the staircase limit) to values closer to one (close to the staircase limit) around the value $L_{R h} / L_{\varepsilon} \approx 6$. 

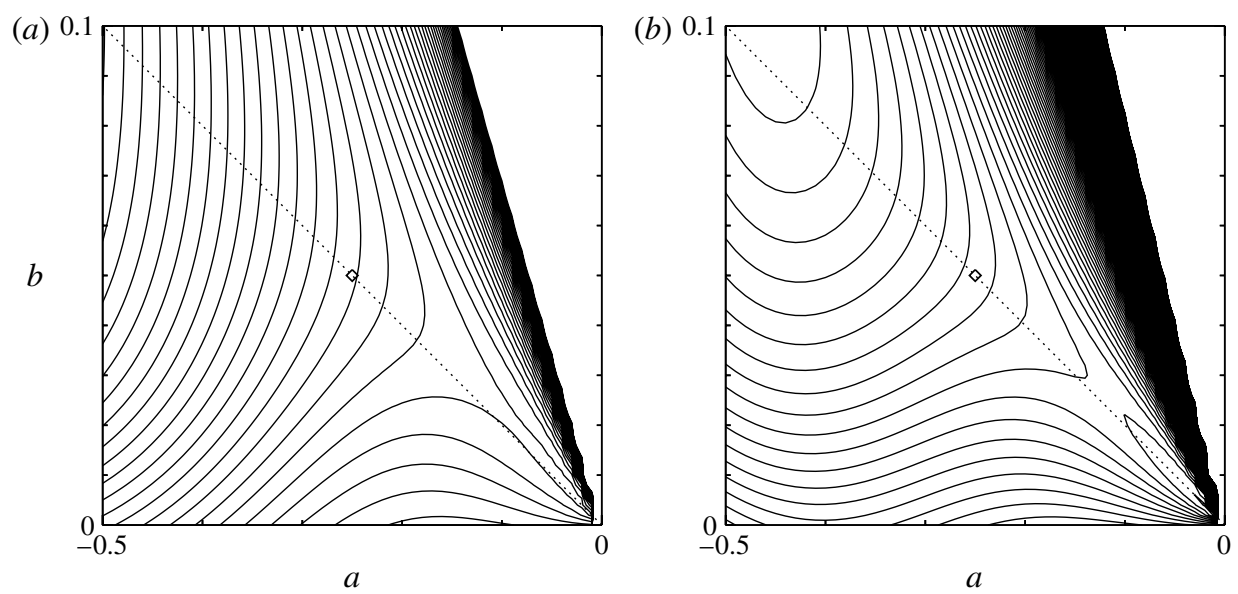

FIGURE 14. Chi-square goodness of fit of the staircase measures $1-I_{1} / I_{2}(a)$ and $I_{3} / I_{2}(b)$ to a cubic in the quantity $r^{a} \varepsilon^{b}$ for exponent $a$ in the range $[-0.5,0]$ and $b$ in the range $[0,0.1]$. Dotted line indicates the relation $a=-5 b$, while the point $a=-0.25, b=0.05$, for which $r^{a} \varepsilon^{b}$ is equivalent to the quantity $L_{R h} / L_{\varepsilon}$ is indicated by the diamond. Contour interval is 0.005 and 0.002 , with minimum values in the upper right corner of 0.080 and 0.117 , for $a$ and $b$ respectively.

Although there is some variance, the extent to which the data cluster around a well-defined line is striking. It should be noted that this collapse is obtained despite large variance in details such as the spacing of individual jets obtained for different forcing types or even for different realizations of the random forcing for identical forcing parameters. Note that the irregularity of the staircases obtained with broadband forcing (whether in physical or spectral space) means that any attempt to fit profiles of $q\left(y_{e}\right)$ to a regular notional staircase would fail. From our analysis it appears that regularly spaced jets will only be obtained in the particular cases where the forcing has a well-defined peak at some dominant wavenumber.

While there is a clear clustering of the data points around a well-defined curve, it could nevertheless be questioned whether other combinations of physical parameters lead to still better collapse. To test this we have plotted the two measures $1-I_{1} / I_{2}$ and $I_{3} / I_{2}$ against the combination $r^{a} \varepsilon^{b}$ for a variety of values of the exponents $a$ and $b$. To test how well the data collapse onto a curve, for each value of $a$ and $b$, we computed the least-squares fit to a low-degree polynomial in the quantity $r^{a} \varepsilon^{b}$; this choice is somewhat arbitrary, but has the property of being a smooth curve that has enough degrees of freedom to represent the approximate shape of the data clusters shown in figure 13. We note, in particular, that there is no reason to suppose a direct power law relation that would motivate fitting to a straight line.

We measure the degree of collapse to a polynomial by the chi-square goodness of fit. For the case of a cubic polynomial, this quantity is shown in figure 14 for the two measures $1-I_{1} / I_{2}$ and $I_{3} / I_{2}$. There are two features worth noting. First, the best fit to a cubic is obtained for values of $a$ and $b$ larger (in magnitude) than the values $a=-0.25, b=0.05$ (represented by the diamond) that are equivalent to the quantity $L_{R h} / L_{\varepsilon}$; the minimum values are 0.080 and 0.117 for $1-I_{1} / I_{2}$ and $I_{3} / I_{2}$, respectively. Second, a valley in the chi-square goodness of fit runs up the line $a=-5 b$ (dotted), indicating that the best collapse of the data is obtained for a ratio of exponents 
similar to the ratio of exponents in the quantity $L_{R h} / L_{\varepsilon}$. That the overall minimum occurs at higher values of $a$ and $b$ may indicate simply that the choice of cubic is not the optimal shape curve for fitting. Indeed, fitting to a quadratic instead of a cubic, yields a similar valley aligned along $a=-5 b$, but with the overall minimum on the other side of the diamond, i.e. for values of $a$ and $b$ smaller in magnitude than the values $a=-0.25, b=0.05$. Fitting to a quartic gives a minimum that is close to $(-0.25,0.05)$, but the value of the minimum is larger than for the case of the cubic and quadratic (which have similar minimum values). It thus appears that the objective 'best' exponents depend upon the functional form assumed for the dependence of $1-I_{1} / I_{2}$ and $I_{3} / I_{2}$ on $r^{a} \varepsilon^{b}$. This is further supported by the observation that the chi-square goodness of fit is rather flat over a wide range containing the point $(-0.25,0.05)$.

\section{Conclusions}

The aim of this paper was to examine the circumstances under which the equilibrium state of forced geostrophic turbulence might approach the staircase limit of perfectly homogenized zones of potential vorticity separated by sharp fronts, as studied recently by Dritschel \& McIntyre (2008) and Dunkerton \& Scott (2008). The question is of interest in part because of the existence of the simple analytic result relating jet separation to jet strength in the staircase limit, as reviewed in $\$ 2$. More importantly, however, it is of interest because the steepness of potential vorticity gradients in jet cores is expected to play a key role in governing lateral transport across the jets. The latter issue is relevant to questions concerning, for example, the transport of chemical species between the troposphere and stratosphere across the subtropical jet, or across the winter stratospheric polar vortex edge, as well as the lateral transport of heat, momentum, and salinity in the oceans. Understanding the physical parameters that control potential vorticity gradients in jet cores is therefore central to understanding many aspects of the global atmospheric and oceanic circulations.

The numerical experiments reported in $\S 4$ illustrate that the approach to a staircaselike distribution of potential vorticity can be described effectively by a single nondimensional parameter, expressible as the ratio of two familiar length scales $L_{R h} / L_{\varepsilon}$. The simple result is that the staircase limit is approached for large $L_{R h} / L_{\varepsilon}$. For a variety of forcing types, well-defined staircase distributions (strong jets) are robustly produced for $L_{R h} / L_{\varepsilon} \sim O(10)$, whereas for $L_{R h} / L_{\varepsilon} \sim O(1)$ no clear staircasing occurs. Further, there appears to be no limit to the degree to which the staircase limit is approached: potential vorticity gradients between jets are essentially zero, while gradients in the jet cores are limited only by the numerical discretization. These results have been obtained for three qualitatively different types of forcing mechanism (including the commonly used band-limited spectral-space forcing) suggesting that they are robust to details of the forcing and its spectrum. We note also that we have observed a similar dependence on $L_{R h} / L_{\varepsilon}$ in similar experiments using a standard pseudo-spectral model (not shown), albeit at lower resolution than the results presented here.

The observed dependence on $L_{R h} / L_{\varepsilon}$ appears plausible on simple physical grounds. For a given total energy of the system, strong forcing (large $L_{\varepsilon}$ ) produces eddies with potential vorticity anomalies that are large compared to the largest possible jump across a jet. In this case eddies may interact strongly with jet cores causing mixing across them and preventing potential vorticity gradients from intensifying unhindered. In the weak forcing case, on the other hand, the eddy potential vorticity anomalies are 
always much weaker than the potential vorticity jumps that develop between mixing zones. As was shown in Dritschel \& McIntyre (2008), it is very difficult for an eddy to penetrate a jet whose potential vorticity jump is greater than the eddy intensity. In part, this is because the eddy is not strong enough to withstand the strong shear in the region of the jet core, and so will be strongly deformed and ultimately dissipated by small-scale processes. In part, it is due to the dynamical resilience of the potential vorticity jump itself.

To quantify this, we analysed the maximum vorticity anomalies associated with the jet and eddy part of the vorticity field, making use of the decomposition described in $\S 4.2$. The maximum jet vorticity anomaly increases with $L_{R h} / L_{\varepsilon}$, from around 23 at $L_{R h} / L_{\varepsilon}=3.0$ to around 42 at $L_{R h} / L_{\varepsilon}=10.8$, larger values being associated with larger departures from $\beta y$ for cases closer to the staircase limit. In contrast, the maximum eddy vorticity decreases with $L_{R h} / L_{\varepsilon}$ from around 36 at $L_{R h} / L_{\varepsilon}=3.0$ to 7 at $L_{R h} / L_{\varepsilon}=10.8$. In the case of $L_{R h} / L_{\varepsilon}=10.8$, therefore, the eddy field is far too weak to be able to cause any mixing across the jets, yet is sufficient to maintain the weak gradients in between them. The cross-over point, for which the jet and eddy vorticity maxima are comparable, occurs at a value of $L_{R h} / L_{\varepsilon} \approx 5$, consistent with the onset of strong jets observed in figure 13 .

An important point to note is that, in our view, it is the strength of the forcing and not the strength of the friction which is crucial in controlling the emergence of strong jets. This is in contrast both to the recent work of Berloff et al. (2011), who considered the effect of bottom friction in baroclinically forced turbulence, and to the phenomenological arguments of Vallis (2006), who discussed the influence of a parameter similar to $L_{R h} / L_{\varepsilon}$ on the halting of the inverse cascade by friction. Friction is here only needed to achieve a stationary state, but does not play an active role in controlling the extent of staircase development. Indeed, in variations of the numerical experiments shown here in which friction is absent and in which the energy is allowed to increase linearly, a similar dependence of the jet strength on the instantaneous value of $L_{R h} / L_{\varepsilon}$ is found, which may now be considered as depending on $\beta, \varepsilon$, and the total energy $\mathscr{E}$ (a similar conclusion was found in a study of undamped topographic forcing by Scott $\&$ Tissier 2012).

An interesting additional feature of the experiments reported above is the presence on the jets of persistent waves with $O(1)$ slopes, even in cases of extremely weak forcing. Preliminary analysis suggests that these waves arise in such a way as to maintain the flow in a state of marginal stability in the presence of a potential vorticity distribution that is weakly non-monotonic in the north-south direction. A full analysis of these waves and the origin of weakly non-monotonic potential vorticity will be considered in future work.

The model used in the present study is arguably the simplest possible model that captures the two main dynamical processes involved: turbulent mixing and Rossby wave propagation. The results should therefore be interpreted with appropriate caution. We note that even the relatively simple extension to a finite deformation radius greatly increases the complexity of the problem, since the relative magnitudes of three length scales must be considered. Further, the equilibrium states obtained, typically comprising jets that are highly undular coexisting with strong coherent vortices, are much harder to classify than the relatively straight jets obtained above. Further extensions to three-dimensional quasi-geostrophic flows will additionally require a more careful consideration of the different processes that might act as an eddy forcing (for example, internal baroclinic instability driven by large-scale heating, or convective 
penetration at small scales) and how these should be represented in the model; the latter remains a formidable challenge.

\section{Acknowledgements}

The authors thank M. McIntyre and P. Berloff for helpful comments and discussions. R.K.S. received partial support for this research from the National Science Foundation; D.G.D. received support from a Leverhulme Trust Research Fellowship.

\section{REFERENCES}

Baldwin, M. P., Rhines, P. B., Huang, H.-P. \& McIntyre, M. E. 2007 The jet-stream conundrum. Science 315, 467-468.

Berloff, P., Karabasov, S., Farrar, J. T. \& Kamenkovich, I. 2011 On latency of multiple zonal jets in the oceans. J. Fluid Mech. 686, 534-567.

DRITSCHEL, D. G. 1989 Contour dynamics and contour surgery: numerical algorithms for extended, high-resolution modelling of vortex dynamics in two-dimensional, inviscid, incompressible flows. Comput. Phys. Rep. 10, 78-146.

Dritschel, D. G. \& AMBAUM, M. H. P. 1997 A contour-advective semi-Lagrangian numerical algorithm for simulating fine-scale conservative dynamical fields. Q. J. R. Meteorol. Soc. 123, 1097-1130.

Dritschel, D. G. \& Fontane, J. 2010 The combined Lagrangian advection method. J. Comput. Phys. 229, 5408-5417.

Dritschel, D. G. \& MCIntyre, M. E. 2008 Multiple jets as PV staircases: the Phillips effect and the resilience of eddy-transport barriers. J. Atmos. Sci. 65, 855-874.

Dritschel, D. G. \& SCOTT, R. K. 2009 On the simulation of nearly inviscid two-dimensional turbulence. J. Comput. Phys. 228, 2707-2711.

Dritschel, D. G. \& Scott, R. K. 2011 Jet sharpening by turbulent mixing. Phil. Trans. R. Soc. Lond. A 369, 754-770.

DUnkeRTON, T. J. \& SCOTT, R. K. 2008 A barotropic model of the angular momentum conserving potential vorticity staircase in spherical geometry. J. Atmos. Sci. 65, 1105-1136.

FontANe, J. \& DRITSChel, D. 2009 The HyperCASL algorithm: a new approach to the numerical simulation of geophysical flows. J. Comput. Phys. 228, 6411-6425.

Galperin, B., Nakano, H., Huang, H.-P. \& Sukoriansky, S. 2004 The ubiquitous zonal jets in the atmospheres of giant planets and earth's oceans. Geophys. Res. Lett. 31, L13303.

Huang, H.-P., Galperin, B. \& Sukoriansky, S. 2001 Anisotropic spectra in two-dimensional turbulence on the surface of a rotating sphere. Phys. Fluids 13, 225-240.

Maltrud, M. E. \& VAllis, G. K. 1991 Energy spectra and coherent structures in forced two-dimensional and beta-plane turbulence. J. Fluid Mech. 228, 321-342.

Marcus, P. S. 1993 Jupiter's great red spot and other vortices. Annu. Rev. Astron. Astrophys. 31, 523-573.

MCInTYRe, M. E. 1982 How well do we understand the dynamics of stratospheric warmings?. J. Meteorol. Soc. Japan 60, 37-65, special issue in commemoration of the centennial of the Meteorological Society of Japan, ed. K. Ninomiya.

MCIntyre, M. E. 2008 Potential-vorticity inversion and the wave-turbulence jigsaw: some recent clarifications. Adv. Geosci. 15, 47-56.

PAnetTA, R. L. 1993 Zonal jets in wide baroclinically unstable regions: persistence and scale selection. J. Atmos. Sci. 50, 2073-2106.

Peltier, W. R. \& Stuhne, G. R. 2002 The upscale turbulent cascade: shear layers, cyclones and gas giant bands. In Meteorology at the Millennium (ed. R. Pierce). Academic.

Phillips, O. M. 1972 Turbulence in a strongly stratified fluid - is it unstable? Deep-Sea Res. 19, 79-81.

Rhines, P. B. 1975 Waves and turbulence on a beta-plane. J. Fluid Mech. 69, 417-443.

SAFFMAN, P. G. 1971 On the spectrum and decay of random two-dimensional vorticity distributions at large Reynolds number. Stud. Appl. Maths 50, 377-383. 
SCOTT, R. K. 2010 The structure of zonal jets in shallow water turbulence on the sphere. In IUTAM Symposium on Turbulence in the Atmosphere and Oceans (ed. D. G. Dritschel), IUTAM Bookseries, vol. 28, pp. 243-252. Springer.

Scott, R. K. \& Polvani, L. M. 2007 Forced-dissipative shallow water turbulence on the sphere and the atmospheric circulation of the gas planets. J. Atmos. Sci. 64, 3158-3176.

Scott, R. K. \& TISSIER, A.-S. 2012 The generation of zonal jets by large-scale mixing. Phys. Fluids (submitted).

SukORIAnsky, S., Dikovskaya, N. \& GALPERIN, B. 2007 On the arrest of inverse energy cascade and the Rhines scale. J. Atmos. Sci. 64, 3312-3327.

Vallis, G. K. 2006 Atmospheric and Oceanic Fluid Dynamics. Cambridge University Press.

Williams, G. P. 1978 Planetary circulations: 1. Barotropic representation of Jovian and terrestrial turbulence. J. Atmos. Sci. 35, 1399-1424. 